\title{
Low response to intradermal hepatitis $B$ vaccination in incident hemodialysis patients
}

\begin{abstract}
Authors
Regina H. Medeiros ${ }^{1}$

Ana Elizabeth PL

Figueiredo $^{1}$

Carlos Eduardo

Poli-de-Figueiredo ${ }^{1}$

Domingos Otávio

d'Avila'

Carlos Abaeté de

los Santos ${ }^{1}$

${ }^{1}$ Faculdade de Medicina Pontifícia Universidade Católica do Rio Grande do Sul
\end{abstract}

Submitted on: 05/102010 Accepted on: 07/112010

Corresponding author: Carlos Abaeté de los

Santos

Av. Ipiranga, 6690

Porto Alegre - RS - Brazil

CEP: 90601-010

Phone: 5551 3223-0366

E-mail: abaete@pucrs.br

The present study was conducted at the PostGraduation in Medicine and Health Sciences,

Faculdade de Medicina, Pontifícia Universidade Católica do Rio Grande do Sul. Porto Alegre, RS, Brazil.

The authors declare no conflict of interest was involved in this trial. The study has been registered as a Trial at ANZCTR.org. au under study number ACTRN12609000616279.

\section{Abstract}

Introduction: Hepatitis B (HB) may progress to cirrhosis and liver carcinoma. Its prevalence is estimated at $3.2 \%$ in hemodialysis (HD) patients. $\mathrm{HB}$ vaccine when applied intramuscularly (IM) in end-stage renal disease patients often does not induce appropriate antibody titers. However, there has been suggestion for intradermal (ID) to be a more effective inoculation method. Objective: To compare the immune response to IM or ID vaccine administration on HD patients. Patients and Methods: Thirty one incident HD patients were randomly assigned alternately to IM or ID vaccine inoculation. Vaccine doses were applied at three monthly intervals, with patients being followed-up for six months. Sixteen patients were assigned to IM (40 $\mathrm{mg} /$ dose) and 15 to ID (4 mg/dose) vaccine administration. HB-virus surface antibody titer, hematimetric parameters, serum urea level and $\mathrm{Kt} / \mathrm{V}$ were monthly evaluated. C-reactive protein, parathormone, ferritin, aminotransferases and albumin serum levels were evaluated before and at the sixth month of the initial inoculation. Results: Urea levels were significantly higher in the ID group $(\mathrm{P}(1)=0.031)$; ferritin levels were higher in the IM $(\mathrm{P}(2)=0.037)$ and C-reactive protein levels tended to be higher in the ID group. An interim evaluation by the Safety Monitoring Committee recommended discontinuing the study as IM vaccination had converted $62.5 \%$ of the exposed subjects, while ID inoculation converted only $13.3 \%$. Conclusion: As performed, ID applied vaccine was inferior to the IM inoculation. Such result may depend on the inoculated doses or some other factor, such as inflammation.

Keywords: hepatitis B, immunization, chronic kidney failure.

[J Bras Nefrol 2011;33(1): 29-33] CElsevier Editora Ltda.

\section{INTRODUCTION}

Chronic Hepatitis B (HB) infection may progress to cirrhosis and liver carcinoma. Incidence of $\mathrm{HB}$ among hemodialysis (HD) patients has been steadily and progressively decreasing, after strict vaccination policies were adopted. ${ }^{1}$ Yet a recent survey has estimated the prevalence of $\mathrm{HB}$ among Brazilian HD patients at 3.2\%. ${ }^{2}$

Recommendations are for every nonimmunized patient starting HD to be vaccinated. However, $\mathrm{HB}$ vaccine conversion rates are low in end-stage renal disease (ESRD) patients undergoing HD - only $43 \%$ to $66 \%$ of patients achieve adequate antibody titers, compared with more than $95 \%$ of healthy subjects. ${ }^{3,4}$ The impaired response to vaccination seems to depend on multiple factors, such as age, nutritional condition, presence of inflammation and decrease in erythropoietin levels, besides lower $\mathrm{T}$ and $\mathrm{B}$ lymphocytes activity. ${ }^{5}$ The immune response induced by IM or ID HB vaccination was found to be different when comparing the groups. ${ }^{6}$

The aim of the current study was to compare the responses to IM or ID HB vaccine inoculation in non-immunized incident HD patients.

\section{Patients and Methods}

This was a randomized cohort study that enrolled alternatively incident HD patients before reaching the third month of treatment to compare the immune response to either IM or ID inoculation of $\mathrm{HB}$ vaccine. The study protocol was approved by the University Research Ethics Committee and a written consent was obtained from each subject before enrollment. 
Thirty-one patients were enrolled alternatively - 16 individuals were allocated to receive the vaccine intramuscularly (IM) and 15 to receive it by intradermal inoculation (ID). Diabetes mellitus was the cause of ESRD in four subjects of the IM group and in three of the ID; hypertension in two of each group; glomerular disease in one of the IM, Vesicoureteral reflux in one of each group; polycystic disease in one of the ID, while an unknown cause occurred once in each group. Individuals allocated to the IM group were inoculated with Engerixâ vaccine, from GlaxoSmithKline Lab, England (40mg/ dose/monthly, for three months into a deltoid muscle. The ID group received an intradermal inoculation $(4 \mathrm{mg} / \mathrm{dose})$ at the internal side of the forearm, at equal time intervals. The vaccination schedule and dosage were precviously suggested by the Brazilian Ministry of Health. ${ }^{7}$ All the participants tested negatively for human acquired immunodeficiency and hepatitis $C$ viruses. None had been previously vaccinated for $\mathrm{HB}$ or had significant hepatitis B-surface antigen antibody (Anti-HBs) titers. All the enrolled patients were kept on the same HD prescription - four-hour, thrice-weekly, standard bicarbonate, polysulfone dialyzers, using a Fresenius 4008 B dialysis machine (Fresenius Medical Care, Bad Homburg, Germany) with dialysate flow maintained at $500 \mathrm{~mL} / \mathrm{min}$, blood flow in the range of 250 to $300 \mathrm{~mL} / \mathrm{min}$, and unfractionated heparin as standard anticoagulation. All patients, except ten utilizing a central catheter, were using an arteriovenous fistula as vascular access.

Demographic and clinical variables - age, gender; cardiovascular disease, cancer, infection, HD vintage, use of fistula or central catheter access - were analyzed. Therapeutic and laboratory variables: number of transfusions, erythropoietin dose, $\mathrm{Kt} / \mathrm{V}$, serum urea, red blood cell count and Anti-HBs titers were monthly analyzed, while others, such as C-reactive protein (CRP), ferritin, parathyroid hormone (PTH), alanine-aminotransferase, (ALT), aspartate-aminotransferase (AST), and albumin were measured initially and at the six-month interval.

Yet an interim evaluation, carried out by the Safety Monitoring Committee, at a three-month interval, recommended discontinuing the study as the antibody titers in a majority of the ID group patients did not reach protective levels (less than 10 $\mathrm{mIU} / \mathrm{mL}$ ) and were significantly lower than those on the IM group. Consequently, only seventeen patients were evaluated: nine in the IM and eight in the ID group. Total follow-up time was 6 months.
Data are presented as mean and standard deviation (SD), median and interquartile range (IQR), or percentage. Student $t$ test or Mann-Whitney $U$ test was used to compare two continuous variables; chisquare $\left(\mathrm{c}^{2}\right)$ - or Fisher exact test - was used in comparisons between categorical variables. Repeatedmeasure ANOVA was also used. ( $\mathrm{p}^{(1)}$ regards differences between groups at the beginning of the study; $\mathrm{p}^{(2)}$, changes over time per group; $\mathrm{p}^{(3)}$ addresses differences over time and between groups; $\mathrm{P}^{(4)}$, differences at the groups' mean time-point. The Statistical Package for Social Sciences, (SPSS version 11.5 for Windows, SPSS Inc., Chicago, IL, USA) was used in all statistical analysis. A value of $\mathrm{p} \leq 0.05$ was considered significant.

\section{Results}

Thirty-one patients were included; 16 randomized for the IM and 15 for the ID group. Due to the study deferment, only 17 individuals completed the protocol: nine in the IM group and eight in the ID group. No significant age difference between groups was observed $(59 \pm 17$ vs. $57.0 \pm 17.0$ years; $\mathrm{p}=0.638$, for IM and ID group, respectively). The male to female ratio was $5 / 4$ in the IM and $6 / 2$ in the ID group $(\mathrm{p}=$ $0.347)$. Time on dialysis was $2.2 \pm 1.2$ in IM and 2.3 \pm 1.4 months in ID $(\mathrm{p}=0.864)$. A central catheter was used by four individuals in the IM and by six in ID group $(\mathrm{p}=0.353)$. Half of patients in both groups required at least one blood transfusion. Bacterial infection was detected in two patients on each group. Erythropoietin was used in the same proportion in both groups.

Table 1 shows results of laboratory tests during the study. Blood titers of Anti-HBs and serum urea levels were significantly different between groups at the beginning of the study $(\mathrm{p}=0.031)$. Only AntiHBs, hematocrit, hemoglobin and ferritin titers were significantly different during the analysis period $\left(\mathrm{p}^{(2)}\right.$ $<0.001, \mathrm{p}^{(2)}=0.001, \mathrm{p}^{(2)}=0.004, \mathrm{p}^{(2)}=0.037$, respectively). Additionally, only Anti-HBs titers were significantly different $\left(\mathrm{p}^{(3)}<0.001\right)$ among groups. Finally, only Anti-HBs titer and urea levels significantly differed $\left(\mathrm{p}^{(4)}<0.001\right.$ and $\mathrm{p}^{(4)}=0.035$, respectively). In the IM group, $10(62.5 \%)$ patients achieved protective titers of Anti-HBs ( $\left.{ }^{3} 10 \mathrm{UI} / \mathrm{mL}\right)$, while only two $(13.3 \%)$ patients in ID group reached acceptable titer. The evolution of Anti-HBs titers along the observation period, per group, is shown in the Figure 1. The responses significantly differed from the third to the sixth month. 
Table 1 LABORATORY DETERMINATIONS ( $N=17)$

\begin{tabular}{|c|c|c|c|c|c|c|c|c|}
\hline \multirow[b]{2}{*}{ Variable } & \multicolumn{2}{|c|}{ Intramuscular $(\mathrm{n}=9)$} & \multicolumn{2}{|c|}{ Intradermic $(n=8)$} & \multirow[b]{2}{*}{$\mathrm{p}^{(1)}$} & \multirow[b]{2}{*}{$\mathrm{p}^{(2)}$} & \multirow[b]{2}{*}{$p^{(3)}$} & \multirow[b]{2}{*}{$\mathrm{p}^{(4)}$} \\
\hline & Initial & 6 mounth & Initial & 6 mounth & & & & \\
\hline Albumina (mg/dL) & $3.7 \pm 0.2$ & $3.8 \pm 0.3$ & $3.6 \pm 0.4$ & $3.9 \pm 0.8$ & 0.867 & 0.376 & 0.659 & 0.867 \\
\hline ALT (U/I) & $22 \pm 6$ & $23 \pm 9$ & $18 \pm 6$ & $20 \pm 8$ & 0.290 & 0.433 & 0.608 & 0.290 \\
\hline Anti-HBs (UI/mL) & $2(2-3)$ & $248(5-1000)$ & $2(1-2)$ & $2(1-22)$ & 0.423 & $<0.001$ & $<0.001$ & $<0.001$ \\
\hline AST (U/I) & $18 \pm 6$ & $23 \pm 1$ & $14 \pm 9$ & $16 \pm 9$ & 0.090 & 0.131 & 0.522 & 0.090 \\
\hline Ferritin $(\mu \mathrm{g} / \mathrm{dL})$ & 301 (36-1115) & 598 (323-1484) & $198(114-651)$ & $444(135-844)$ & 0.139 & 0.037 & 0.733 & 0.212 \\
\hline $\mathrm{Hb}(\mathrm{mg} / \mathrm{dL})$ & $7.7 \pm 2.0$ & $11.3 \pm 2.3$ & $8.3 \pm 1.5$ & $9.7 \pm 2.0$ & 0.253 & 0.001 & 0.089 & 0.482 \\
\hline $\mathrm{Ht}(\%)$ & $24 \pm 6$ & $33 \pm 6$ & $27 \pm 5$ & $31 \pm 6$ & 0.805 & 0.004 & 0.187 & 0.792 \\
\hline $\mathrm{Kt} / \mathrm{N}$ & $1.31 \pm 0.20$ & $1.20 \pm 0.10$ & $1.20 \pm 0.30$ & $1.30 \pm 0.50$ & 0.395 & 0.736 & 0.132 & 0.810 \\
\hline 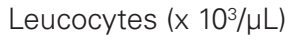 & $8.3 \pm 3.5$ & $6.7 \pm 1.2$ & $7.8 \pm 2.6$ & $7.7 \pm 1.5$ & 0.084 & 0.311 & 0.370 & 0.748 \\
\hline $\mathrm{CRP}(\mathrm{mg} / \mathrm{dL})$ & $2.8(0.17-11.2)$ & $0.4(0.3-6.0)$ & $4.4(0.3-11.5)$ & $5.5(0.2-19.4)$ & 0.541 & 0.482 & 0.226 & 0.411 \\
\hline Urea (mg/dL) & $124 \pm 22$ & $122 \pm 34$ & $165 \pm 100$ & $150 \pm 52$ & 0.031 & 0.523 & 0.636 & 0.035 \\
\hline
\end{tabular}

Data are presented as mean \pm standard deviation or median (interquartile range). $p(1)$ : Student's t test or Mann-Whitney $\mathrm{U}$ test for initial differences between groups; $p(2)$ Repeated measures ANOVA for same group changes over time; $p(3)$ : Between groups over time; $p(4)$ : Between groups at study's mean point; Anti-HBs: Hepatitis B surface antibody; ALT: Alanine-transferase; AST: Aspartate-transferase; Hb: Hemoglobin; Ht: Hematocrit; Kt/N: Normalized dialysis clearance; CRP: C-reactive protein.

Figure 1. Immune response to intramuscular or intradermal vaccine inoculation.

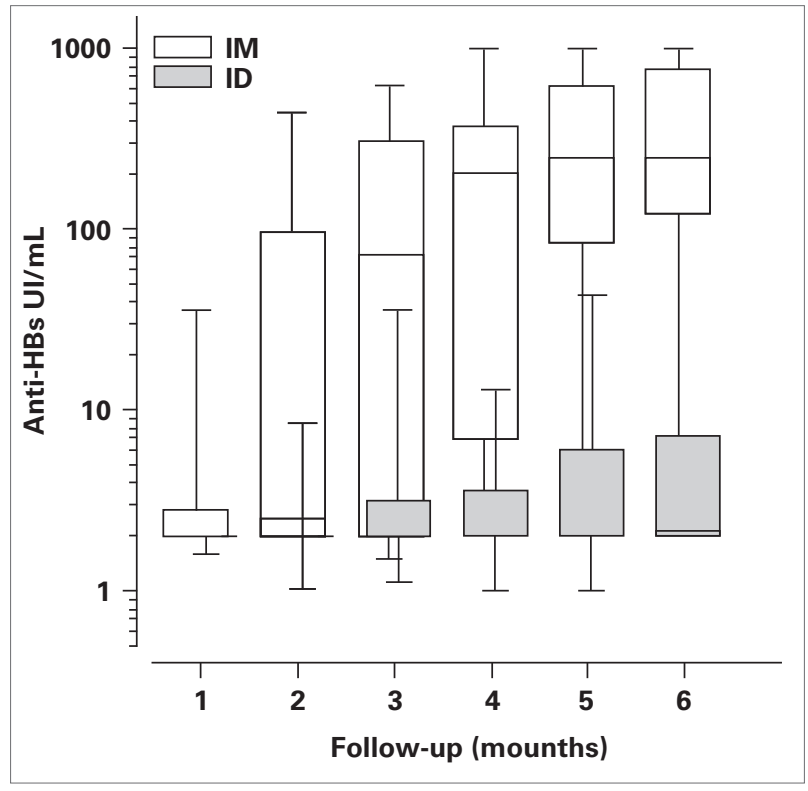

Reprinted from Medeiros et al. Intramuscular or intradermal hepatitis B vaccine administration in hemodialysis patients? Am J Kidney Dis 2009; 54:981-982, with permission from Elsevier.

\section{Discussion}

The elicited anti-HBs titers were significantly different in response to HB vaccine administration by IM or by ID route. The effect favoring the IM group was evidenced early in the course of the study. After an interim analysis, the Safety Monitoring Committee suggested interrupting the trial. The IM vaccine administration immunized almost two-thirds of the exposed patients, a result similar to that obtained in healthy populations. ${ }^{8,9}$ Yet the ID administration was effective in only a minority of the inoculated individuals. The results are at substantial discrepancy with previous studies. ${ }^{10,11,12}$ However, a previous study comparing IM ministration with ID inoculation of the vaccine demonstrated similar results. ${ }^{13}$ Yet the aggregate ID antigen dose was higher than that devised for the current study. Also, intradermal inoculation was effective in $94 \%$, applying a larger vaccine dose $(20 \mathrm{mg}$ ) at shorter time intervals (6 doses/week). ${ }^{14}$ Different possibilities might be considered to explain the disparate response observed: diverse clinical conditions or population characteristics, or different vaccine doses. Only incident HD patients were included in the current one - all patients were naïve in regards to $\mathrm{HB}$ vaccine. Even though initial Anti- HBs titers were always lower than $10 \mathrm{UI} / \mathrm{mL}$, they were significantly higher in the IM group. The ID group patients received a smaller vaccine dose and this might be one of the limiting factors of the study. This group also had higher urea levels than the IM, at the beginning, remaining stable towards the end of the study. It has been well known that ESRD individuals have lower immunological responses- humoral and cellular- dysfunctional monocytes and polymorphs, besides higher levels of interleukin-1 and tumor necrosis factor. ${ }^{5,15}$ The significance of such data is not clear, since lower quantities of dialysis might be responsible for the observed differences. ${ }^{16}$ Yet a measure of dialysis efficiency - the Kt/V - did not reveal differences between 
groups. Hemoglobin and hematocrit determinations were lower in IM than in the ID group at the beginning. A recent study suggested that patients with lower hemoglobin levels may have weaker immunologic response to vaccines. ${ }^{8,15}$ Nevertheless, the IM group showed an average hemoglobin determination higher than $11.0 \mathrm{~g} / \mathrm{dL}$ at the end of the study, while the ID did not reach such a level. Even if not significantly different, increased levels of CRP, ferritin and a higher number of patients using a central venous catheter as vascular access in the ID group might imply a higher inflammatory status - red blood cell counts may be lower in presence of inflammation. ${ }^{17}$

Vaccine administered by the ID was less effective than by the IM route. Nevertheless, lower doses of ID vaccine have been effectively used in healthy subjects. Additionally, it should not be overlooked that adequate immune responses were induced by higher doses of ID vaccine, or with more frequent doses. ${ }^{9}$ However, a majority of studies employed low ID vaccine doses. ${ }^{4,10,11}$ A recent study demonstrated revaccination higher conversion rates, using the ID route, in stable HD patients not responding to previous IM vaccination. ${ }^{18}$ The current study is contrary to those findings. ${ }^{19}$ Such a difference might be attributed to revaccination, against initial immunization, prevalent versus incident HD individuals, patients' clinical condition, characteristics of the population at risk or, perhaps, different vaccine application intervals and doses. Inflammation might also have been a significant confounding factor in the vaccine immune response ${ }^{20}$. Contrarily to previous studies that assessed stable patients for a longer treatment period, the current cohort was composed of incident patients. It is not unconceivable that the early interruption of the study blunted potential later responses. It is also possible that the reduced response to ID inoculation was due to multiple factors, including the occurrence of inflammation.

In conclusion, monthly administration of ID HB vaccine to incident $H D$ patients was significantly less effective than IM inoculation.

\section{AcKNOWLEDgeMENTS}

Special acknowledgements to Carlos E. Poli-de Figueiredo is a CNPq Researcher.

\section{References}

1. Mioli VA, Balestra E, Bibiano L et al. Epidemiology of viral hepatitis in dialysis centers: a national survey. Nephron 1992; 61:278-83.
2. Romäo JE Jr, Pinto SWL,Canziani ME, Praxedes JN, Santello JL, Moreira JCM. Censo SBN 2002: informações epidemiológicas das unidades de diálise do Brasil. Braz J Nephrol 2003; 25:187-98.

3. Hassan K, Shternberg L, Alhaj M et al. The effect of erythropoietin therapy and hemoglobin levels on the immune response to Engerix-B vaccination in chronic kidney disease. Ren Fail 2003; 25:471-8.

4. Ghabouli MJ, Sabouri AH, Shoeibi N, Bajestan SN, Baradaran $\mathrm{H}$. High seroprotection rate induced by intradermal administration of a recombinant hepatitis $B$ vaccine in young healthy adults: comparison with standard intramuscular vaccination. Eur J Epidemiol 2004; 19:871-5.

5. Stachowski J, Pollok M, Barth C, Maciejewski J, Baldamus CA. Non-responsiveness to hepatitis B vaccination in haemodialysis patients: association with impaired TCR/CD3 antigen receptor expression regulating co-stimulatory processes in antigen presentation and recognition. Nephrol Dial Transplant 1994; 9:144-52.

6. Rahman F, Dahmen A, Herzog-Hauff S, Bocher WO, Galle PR. Cellular and humoral immune responses induced by intradermal or intramuscular vaccination with the major hepatitis B surface antigen. Hepatology 2000; 31:521-7.

7. Aranda C, Rocha C, Renoiner E, Campos J, Carvalho M. Manual de procedimentos para vacinação. Brasília: Ministério da Saúde/FUNASA; 2001 [http://dtr2001. saude.gov.br].

8. McMaster KR 3rd, Roper JK, Carter JB. Intradermal hepatitis $\mathrm{B}$ vaccination in a 300 -bed primary care hospital: experience with a recombinant vaccine in a fourdose schedule. Am J Infect Control 1993; 21:283-8.

9. Baldy JL, Elisbão MC, Anzai ET et al. Intradermal vaccination of adults with three low doses $(2 \mathrm{mg})$ of recombinant hepatitis B vaccine. I. Seroconversion rate and adverse effects. Mem Inst Oswaldo Cruz 2003; 98:1101-7.

10. Fabrizi F, Andrulli S, Bacchini G, Corti M, Locatelli F. Intradermal versus intramuscular hepatitis b re-vaccination in non-responsive chronic dialysis patients: a prospective randomized study with cost-effectiveness evaluation. Nephrol Dial Transplant 1997; 12:1204-11.

11. Chang PC, Schrander-van der Meer AM, van Dorp WT, van Leer E. Intracutaneous versus intramuscular hepatitis B vaccination in primary non-responding haemodialysis patients. Nephrol Dial Transplant 1996; 11:191-3.

12. Charest AF, McDougall J, Goldstein MB. A randomized comparison of intradermal and intramuscular vaccination against hepatitis B virus in incident chronic hemodialysis patients. Am J Kidney Dis 2000; 36:976-82.

13. Somboonsilp W, Eiam-Ong S, Tungsanga K, Tirawatanapong T. Immune response of intradermal hepatitis B vaccination at lower dose versus intramuscular vaccination at double standard dose in predialytic chronic renal failure patients. J Med Assoc Thai 2003; 86:1122-7.

14. Propst T, Propst A, Lhotta K, Vogel W, Konig P. Reinforced intradermal hepatitis $B$ vaccination in hemodialysis patients is superior in antibody response to intramuscular or subcutaneous vaccination. Am J Kidney Dis 1998; 32:1041-5. 
15. Vlassopoulos D. Recombinant hepatitis B vaccination in renal failure patients. Curr Pharm Biotechnol 2003; 4:141-51.

16. Kovacik V, Sain M, Vukman V. Efficient hemodialysis improves the response to hepatitis $\mathrm{B}$ virus vaccination. Intervirology 2002; 45:172-6.

17. Andrews NC. Disorders of iron metabolism. N Engl J Med 1999; 341:1986-9.

18. Barraclough KA, Wiggins KJ, Hawley CM. Intradermal versus intramuscular hepatitis B vaccination in hemodialysis patients: a prospective open- label randomized controlled trial in nonresponders to primary vaccination. Am J Kidney Dis 2009; 54:95-103.
19. Medeiros RH, Figueiredo AE, Poli-de-Figueiredo CE, d'Avila DO, de los Santos CA. Intramuscular or intradermal hepatitis $\mathrm{B}$ vaccine administration in hemodialysis patients? [letter]. Am J Kidney Dis 2009; 54:981-2.

20. Barraclough KA, Hawley CM, Playford E.G. In reply to "Intramuscular or intradermal hepatitis $\mathrm{B}$ vaccine administration in hemodialysis patients?" [letter] Am J Kidney Dis. 2009;54:982. 